\title{
Estimation of temporally aggregated multivariate GARCH models
}

\author{
Christian M. Hafner ${ }^{1}$ Jeroen V.K. Rombouts ${ }^{2}$ \\ Econometric Institute Report EI 2004-30
}

\begin{abstract}
This paper investigates the performance of quasi maximum likelihood (QML) and nonlinear least squares (NLS) estimation applied to temporally aggregated GARCH models. Since these are known to be only weak GARCH, the conditional variance of the aggregated process is in general not known. Thus, one major condition that is often used in proving the consistency of QML, the correct specification of the first two moments, is absent. Indeed, our results suggest that QML is not consistent, with a substantial bias if both the initial degree of persistence and the aggregation level are high. In other cases, QML might be taken as an approximation with only a small bias. Based on results for univariate GARCH models, NLS is likely to be consistent, although inefficient, for weak GARCH models. Our simulation study reveals that NLS does not reduce the bias of QML in considerably large samples. As the variation of NLS estimates is much higher than that of QML, one would clearly prefer QML in most practical situations. An empirical example illustrates some of the results.
\end{abstract}

Keywords: Multivariate GARCH, temporal aggregation, weak GARCH JEL Classification: C14, C22.

\footnotetext{
${ }^{1}$ Econometric Institute, Erasmus University Rotterdam, P.O.B. 1738, 3000 DR Rotterdam, The Netherlands, chafner@few.eur.nl (corresponding author)

${ }^{2}$ Institut d'Economie Appliqué, HEC Montréal, Canada; CORE, Université catholique de Louvain, Louvain-la-Neuve, Belgium
} 


\section{Introduction}

When estimating parameters of the conditional mean and variance in time series models with conditional heteroskedasticity, one of the conditions for consistency of quasi maximum likelihood estimators (QMLE) is that the first two conditional moments are correctly specified, see e.g. Bollerslev and Wooldridge (1992). If this condition does not hold, then the behavior of QMLE is in general not known. It then depends on the particular model whether the bias of QMLE is substantial or negligible.

In the GARCH context, Drost and Nijman (1993) have shown that temporally aggregated GARCH processes are only weak GARCH, in the sense that what is taken to be the conditional variance in the QML estimation is, in reality, just the best linear predictor in terms of a constant and lagged squared errors. Weak GARCH models are closed under temporal aggregation, whereas classical GARCH models are not. Recently, Hafner (2004) has shown that this carries over in a straightforward way to the multivariate case. Thus, estimation of temporally aggregated (univariate or multivariate) GARCH models by QML is in general not consistent.

In the univariate case, there is conflicting evidence on the importance of the bias of QMLE. While Drost and Nijman (1993) report them to be small and negligible, Meddahi and Renault (2004) find substantial biases. The latter authors emphasize that these biases are found in particular in the case of a highly persistent high frequency process and a large aggregation level. This is relevant for financial returns, where the high frequency process is often found to be close to an integrated GARCH (IGARCH) process and aggregation levels can be high, e.g. if weekly or monthly returns are investigated.

The objective of this paper is to extend the simulation results of Drost and Nijman (1993) and Meddahi and Renault (2004) to the multivariate GARCH (MGARCH) case. We want to shed light on the empirically relevant question how important the bias of QML estimation is for alternative aggregation levels. Extending the proposal of Francq and Zakoian (2000) to a multivariate framework, we also consider nonlinear least squares (NLS) estimation that is not efficient but consistent. In our simulation study we evaluate the empirical performance of both estimation procedures under typical bivariate example processes exhibiting high persistence. We also allow for alternative unconditional correlations between the two series. The main results indicate that the bias of QML is negligible

for small aggregation levels but becomes somewhat more pronounced for high levels. The results show that for our sample size, NLS estimation does not reduce the mean square 
error of QML. Considering one-step forecasts of the conditional variances and covariances, QML still has a smaller mean square prediction error than NLS. One would therefore prefer to use QML in a comparable situation in practice.

In an empirical application we show that fitting a multivariate $\operatorname{GARCH}(1,1)$ model for daily DOW JONES/NASDAQ returns is not consistent with fitting the same model at the weekly and bi-weekly frequency. This confirms previous results of Andersen and Bollerslev (1997) in the univariate context, namely that high frequency financial returns do not obey the aggregation schemes of simple $\operatorname{GARCH}(1,1)$ models.

The rest of this paper is organised as follows. Section 2 introduces some notation and defines strong and weak multivariate GARCH models. Section 3 explains temporal aggregation of multivariate GARCH models. The estimation of the parameters of the models is subject of Section 4. The results of a simulation exercise are provided in Section 5. An empirical example illustrates the results of this paper in Section 6 . Section 7 concludes.

\section{From strong to weak MGARCH models}

Let $\varepsilon_{t}$ denote a stochastic vector process with $K$ components and $\mathrm{E}\left[\varepsilon_{t} \mid \mathcal{F}_{t-1}\right]=0$ where $\mathcal{F}_{t-1}$ denotes the set of information available until time $t-1$. In the following we define three versions of MGARCH models.

Definition 1 (MGARCH) Let $H_{t}$ be a stochastic positive definite symmetric matrix. Then, an $M G A R C H(p, q)$ process is defined by

$$
\begin{aligned}
\varepsilon_{t} & =H_{t}^{1 / 2} \xi_{t} \\
h_{t} & =\omega+\sum_{i=1}^{q} A_{i} \eta_{t-i}+\sum_{j=1}^{p} B_{j} h_{t-j}
\end{aligned}
$$

where $h_{t}=\operatorname{vech}\left(H_{t}\right), \omega=\operatorname{vech}(\Omega), \eta_{t}=\operatorname{vech}\left(\varepsilon_{t} \varepsilon_{t}^{\prime}\right)$. The parameter matrices $\Omega, A_{i}$, and $B_{j}$, are square of order $N=K(K+1) / 2$. We can write (2) as a $\operatorname{VARMA}(\max (p, q), p)$ representation of $\eta_{t}$,

$$
\eta_{t}=\omega+\sum_{i=1}^{\max (p, q)}\left(A_{i}+B_{i}\right) \eta_{t-i}-\sum_{j=1}^{p} B_{j} u_{t-j}+u_{t}
$$

where $u_{t}=\eta_{t}-h_{t}$ and where $A_{q+1}=\ldots=A_{p}=0$ if $p>q$ and $B_{p+1}=\ldots=B_{q}=0$ if $q>p$. We consider two cases for $u_{t}$ : 
- If $u_{t}$ is a martingale difference sequence, then $\xi_{t}$ is white noise with identity covariance matrix. This implies that $E\left[\varepsilon_{t} \varepsilon_{t}^{\prime} \mid \mathcal{F}_{t-1}\right]=H_{t}$. If $\xi_{t}$ is i.i.d. then the process $\left\{\varepsilon_{t}\right\}$ is called strong MGARCH. Otherwise, it is called semi-strong MGARCH.

- If $u_{t}$ is only a weak white noise, that is, zero mean and uncorrelated, then the process $\left\{\varepsilon_{t}\right\}$ is said to be a weak MGARCH process. This means that $h_{t}$ is the best linear predictor of $\eta_{t}$ in terms of a constant and lagged values of $\eta_{t}$.

This definition includes the VEC, BEKK and factor GARCH type-models, see Bauwens, Laurent, and Rombouts (2003) for a recent survey of multivariate GARCH models. In their definition of weak GARCH models, Drost and Nijman (1993) define $h_{t}$ to be the best linear predictor of $\eta_{t}$ in terms of a constant and lagged values of $\eta_{t}$, but also in terms of lagged values of $\varepsilon_{t}$. However, as shown by Hafner (2004) this is not necessary to close the model under temporal aggregation. Rather, an assumption on the fourth moment structure is needed, such as (10).

In the following we assume that all eigenvalues of the matrix $\sum_{i=1}^{\max (p, q)}\left(A_{i}+B_{i}\right)$ have modulus smaller than one. This ensures the existence of the unconditional covariance matrix of $\varepsilon_{t}, \operatorname{Var}\left(\varepsilon_{t}\right)=\Sigma_{\varepsilon}$, given by

$$
\sigma=\operatorname{vech}\left(\Sigma_{\varepsilon}\right)=\left(I_{N}-\sum_{i=1}^{\max (p, q)}\left(A_{i}+B_{i}\right)\right)^{-1} \omega,
$$

where the $(N \times 1)$ vector $\sigma$ contains the $K$ unconditional variances and the $K(K-1) / 2$ unconditional covariances of $\varepsilon_{t}$. We will also need finiteness of fourth moments of $\varepsilon_{t}$ in order to calculate the autocovariances of the moving average part of (3). Necessary and sufficient conditions for finite fourth moments are provided by Hafner (2003) for the case of spherical distribution of $\xi_{t}$. We assume these conditions to hold in the rest of the paper. Let us denote by $\Sigma_{u}$ the variance matrix of $u_{t}$, i.e., $\Sigma_{u}=\mathrm{E}\left[u_{t} u_{t}^{\prime}\right]$. Also, we will need the following autocovariance matrices :

$$
\begin{aligned}
& \Gamma_{h}(\tau)=\mathrm{E}\left[\left(h_{t}-\sigma\right)\left(h_{t-\tau}-\sigma\right)^{\prime}\right] \\
& \Gamma_{\eta}(\tau)=\mathrm{E}\left[\left(\eta_{t}-\sigma\right)\left(\eta_{t-\tau}-\sigma\right)^{\prime}\right] \\
& \widetilde{\Gamma}_{\eta}(\tau)=\mathrm{E}\left[D_{K}^{+} \operatorname{vec}\left(\varepsilon_{t} \varepsilon_{t-\tau}^{\prime}\right) \operatorname{vec}\left(\varepsilon_{t} \varepsilon_{t-\tau}^{\prime}\right)^{\prime} D_{K}^{+, \prime}\right]
\end{aligned}
$$

with $D_{m}$ denoting the duplication matrix ${ }^{1}$ and $D_{m}^{+}=\left(D_{m}^{\prime} D_{m}\right)^{-1} D_{m}^{\prime}$. For the case of a high frequency strong GARCH process with spherical innovations, Hafner (2003) gives

\footnotetext{
${ }^{1}$ The $\left(m^{2} \times m(m+1) / 2\right)$ duplication matrix $D_{m}$ is defined by the property $\operatorname{vec}(A)=D_{m} \operatorname{vech}(A)$ for any symmetric $(m \times m)$ matrix $A$.
} 
expressions for $\Sigma_{u}, \Gamma_{\eta}(\tau), \Gamma_{h}(0)$ and Hafner (2004) for $\widetilde{\Gamma}_{\eta}(\tau)$. It can easily be shown that $\Gamma_{h}(\tau)=(A+B)^{\tau} \Gamma_{h}(0), \tau \geq 0$. For the calculations in our paper we also use the asssumption of spherical innovations.

\section{Temporal aggregation}

We will only look at the case of flow variables which is certainly the most relevant one in the case of financial variables such as returns. The aggregation of flow variables is characterized by taking the sum of the high frequency process. Denote the process $\varepsilon_{t}$ that is aggregated over $m$ periods by $\left\{\varepsilon_{m t}^{(m)}, t \in Z Z\right\}$ which is then given by

$$
\varepsilon_{m t}^{(m)}=\varepsilon_{m t}+\varepsilon_{m t-1}+\ldots+\varepsilon_{m t-m+1} .
$$

Now denote by $\eta_{m t}^{(m)}=\operatorname{vech}\left(\varepsilon_{m t}^{(m)} \varepsilon_{m t}^{(m) \prime}\right)$ the vector process that contains the squares and cross-products of the aggregated process $\varepsilon_{m t}^{(m)}$. Since for arbitrary vectors $a$ and $b$ of dimension $K, \operatorname{vech}\left(a b^{\prime}\right)+\operatorname{vech}\left(b a^{\prime}\right)=2 D_{K}^{+} \operatorname{vec}\left(a b^{\prime}\right)$, we have

$$
\eta_{m t}^{(m)}=\eta_{m t}+\eta_{m t-1}+\ldots+\eta_{m t-m+1}+w_{m t}^{(m)} .
$$

where, using the lag operator $L^{k} x_{t}=x_{t-k}$,

$$
\left.w_{m t}^{(m)}=2 D_{K}^{+}\left\{\sum_{i=0}^{m-2} L^{i} \operatorname{vec}\left(\varepsilon_{m t} \varepsilon_{m t-1}^{\prime}\right)+\sum_{i=0}^{m-3} L^{i} \operatorname{vec}\left(\varepsilon_{m t} \varepsilon_{m t-2}^{\prime}\right)+\cdots+\operatorname{vec}\left(\varepsilon_{m t} \varepsilon_{m t-m+1}^{\prime}\right)\right\}\right\} .
$$

Each term of $w_{m t}^{(m)}$ has expectation zero. By further assuming

$$
E\left[\operatorname{vec}\left(\varepsilon_{t} \varepsilon_{t-i}^{\prime}\right) \operatorname{vec}\left(\varepsilon_{t} \varepsilon_{t-j}^{\prime}\right)^{\prime}\right]=0 \quad \forall i, j \geq 0, i \neq j,
$$

and using the martingale difference property of $\varepsilon_{t}$, every term of $w_{m t}^{(m)}$ is uncorrelated with every other term of $w_{m t}^{(m)}$ and with $\eta_{m t-k}, k=0, \ldots, m-1$. It can be shown that condition (10) holds under the stronger assumption of a strong multivariate GARCH process with spherical innovations $\xi_{t}$, see also Hafner (2004).

The variance matrix of $w_{m t}^{(m)}, \Sigma_{w}^{(m)}$ say, is given by

$$
\Sigma_{w}^{(m)}=4 \sum_{i=1}^{m-1}(m-i) \widetilde{\Gamma}(i)
$$

where $\widetilde{\Gamma}(i)$ is given by $(7)$.

To get a better grasp of the matrix notation and the ideas of temporal aggregation we give an illustration for the simplest case by taking $m=2$. 
Example $\mathbf{1}(\mathbf{m}=\mathbf{2}, \mathbf{K}=\mathbf{2})$ The aggregated process is then given by

$$
\begin{aligned}
& \varepsilon_{2 t}^{(2)}=\varepsilon_{2 t}+\varepsilon_{2 t-1} \\
& \varepsilon_{2(t-1)}^{(2)}=\varepsilon_{2(t-1)}+\varepsilon_{2(t-1)-1}=\varepsilon_{2 t-2}+\varepsilon_{2 t-3} \\
& \varepsilon_{2(t-2)}^{(2)}=\varepsilon_{2(t-2)}+\varepsilon_{2(t-2)-1}=\varepsilon_{2 t-4}+\varepsilon_{2 t-5} .
\end{aligned}
$$

Therefore, the new process is a result of adding up the subsequent high frequency vectors $\varepsilon_{t}$ without overlapping. The interest lies in the process $\eta_{2 t}^{(2)}$ which is given by

$$
\begin{aligned}
\eta_{2 t}^{(2)}= & \eta_{2 t}+\eta_{2 t-1}+2 D_{K}^{+} v e c\left(\varepsilon_{2 t} \varepsilon_{2 t-1}^{\prime}\right) \\
\operatorname{vech}\left(\varepsilon_{2 t}+\varepsilon_{2 t-1}\right)\left(\varepsilon_{2 t}+\varepsilon_{2 t-1}\right)^{\prime}= & \left(\begin{array}{c}
\varepsilon_{1,2 t}^{2} \\
\varepsilon_{2,2 t} \varepsilon_{1,2 t} \\
\varepsilon_{2,2 t}^{2}
\end{array}\right)+\left(\begin{array}{c}
\varepsilon_{1,2 t-1}^{2} \\
\varepsilon_{2,2 t-1} \varepsilon_{1,2 t-1} \\
\varepsilon_{2,2 t-1}^{2}
\end{array}\right)+ \\
& \left(\begin{array}{c}
2 \varepsilon_{1,2 t} \varepsilon_{1,2 t-1} \\
\varepsilon_{2,2 t} \varepsilon_{1,2 t-1}+\varepsilon_{1,2 t} \varepsilon_{2,2 t-1} \\
2 \varepsilon_{2,2 t} \varepsilon_{2,2 t-1}
\end{array}\right)
\end{aligned}
$$

To keep the notation simple we focus on multivariate $\operatorname{GARCH}(1,1)$ models in the following. Theorem 1 of Hafner (2004) states that if (10) holds, then the class of weak multivariate $\operatorname{GARCH}(1,1)$ processes is closed under temporal aggregation. This means that the aggregated process $\eta_{m t}^{(m)}$ follows a weak $\operatorname{VARMA}(1,1)$ process on the low frequency time scale that can be written as

$$
\eta_{m t}^{(m)}=\omega^{(m)}+\left(A^{(m)}+B^{(m)}\right) \eta_{m(t-1)}^{(m)}-B^{(m)} u_{m(t-1)}^{(m)}+u_{m t}^{(m)}
$$

where $\omega^{(m)}$ is given by

$$
\omega^{(m)}=m\left(I_{N}+A+B+\ldots+(A+B)^{m-1}\right) \omega
$$

the matrix $B^{(m)}$ is given by the solution to the system of quadratic equations

$$
B^{(m)} G_{m} B^{(m) \prime}+B^{(m)} S_{m}+G_{m}=0,
$$

with all eigenvalues of $B^{(m)}$ smaller than one in modulus, and where the matrices $S_{m}$ and $G_{m}$ are given by

$$
S_{m}=\sum_{i=0}^{2 m-1} J_{i} \Sigma_{u} J_{i}^{\prime}+\Sigma_{w}^{(m)}+(A+B)^{m} \Sigma_{w}^{(m)}\left(A^{\prime}+B^{\prime}\right)^{m}
$$




$$
G_{m}=\sum_{i=0}^{m-1} J_{i+m} \Sigma_{u} J_{i}^{\prime}-(A+B)^{m} \Sigma_{w}^{(m)}
$$

where $S_{m}$ is the variance of $-B^{(m)} u_{m(t-1)}^{(m)}+u_{m t}^{(m)}$, the moving average term in (12), and $G_{m}$ is its first order autocovariance. The matrices $J_{i}$ in (15) and (16) are defined by

$$
\begin{aligned}
& J_{0}=I_{N} \\
& J_{i}=I_{N}+A+(A+B) A+\cdots+(A+B)^{i-1} A, \quad i=1, \ldots, m-1 \\
& J_{m}=\left\{I_{N}+(A+B)+\cdots+(A+B)^{m-2}\right\} A-(A+B)^{m-1} B \\
& J_{i}=\left\{(A+B)^{i-m}+(A+B)^{i-m+1}+\cdots+(A+B)^{m-2}\right\} A-(A+B)^{m-1} B \\
& i=m+1, \ldots, 2 m-2 \\
& J_{2 m-1}=-(A+B)^{m-1} B
\end{aligned}
$$

and $\Sigma_{w}^{(m)}$ is the variance matrix of $w_{m t}^{(m)}$ given in (11).

Note that the solution to (14) is not unique, but restricting $B^{(m)}$ to have eigenvalues smaller than one in modulus provides a unique solution. Although we do not have a proof for this statement, we found it to hold under various scenarios using numerical techniques. Having determined $B^{(m)}$, the coefficient matrix $A^{(m)}$ in (12) is then given by

$$
A^{(m)}=(A+B)^{m}-B^{(m)} .
$$

In the following, let us give an example how these matrices look like in the simple case of $m=2$ and $K=2$.

Example 2 ( $\mathbf{m}=\mathbf{2}$ continued) For simplicity we take an $M G A R C H(1,1)$ process with $K=2$ components $(N=3)$. The autocovariance matrix in (11) becomes

$$
\Sigma_{w}^{(2)}=4 \widetilde{\Gamma}(1)
$$

where $\widetilde{\Gamma}(1)$ in (7) is given in the bivariate case by

$$
\widetilde{\Gamma}(1)=E\left[\begin{array}{lll}
\gamma_{11} & \gamma_{21} & \gamma_{31} \\
\gamma_{21} & \gamma_{22} & \gamma_{32} \\
\gamma_{31} & \gamma_{32} & \gamma_{33}
\end{array}\right]
$$

where

$$
\text { - } \gamma_{11}=\varepsilon_{1, t}^{2} \varepsilon_{1, t-1}^{2}
$$




$$
\begin{aligned}
& -\gamma_{21}=\frac{1}{2} \varepsilon_{1, t} \varepsilon_{2, t} \varepsilon_{1, t-1}^{2}+\frac{1}{2} \varepsilon_{1, t}^{2} \varepsilon_{2, t-1} \varepsilon_{1, t-1} \\
& -\gamma_{31}=\varepsilon_{2, t} \varepsilon_{2, t-1} \varepsilon_{1, t} \varepsilon_{1, t-1} \\
& -\gamma_{22}=\frac{1}{4} \varepsilon_{2, t}^{2} \varepsilon_{1, t-1}^{2}+\frac{1}{2} \varepsilon_{1, t} \varepsilon_{2, t-1} \varepsilon_{2, t} \varepsilon_{1, t-1}+\frac{1}{4} \varepsilon_{1, t}^{2} \varepsilon_{2, t-1}^{2} \\
& -\gamma_{32}=\frac{1}{2} \varepsilon_{2, t}^{2} \varepsilon_{1, t-1} \varepsilon_{2, t-1}+\frac{1}{2} \varepsilon_{1, t} \varepsilon_{2, t-1}^{2} \varepsilon_{2, t} \\
& -\gamma_{33}=\varepsilon_{2, t}^{2} \varepsilon_{2, t-1}^{2}
\end{aligned}
$$

If we suppose $\omega=\left(\omega_{11}, 0, \omega_{33}\right)^{\prime}$ and $A$ and $B(3 \times 3)$ diagonal matrices, then the unconditional covariance is zero, as can easily be seen from (4). If we additionally assume a spherical distribution for $\xi_{t}$ then it can be shown that $E\left(\gamma_{21}\right)=E\left(\gamma_{32}\right)=0$ and that the $(3 \times 3)$ variance matrix of $u_{t}, \Sigma_{u}$, also has zero elements at positions $(2,1),(1,2),(2,3)$ and $(3,2)$. Writing (14) equation-wise, it may be seen that the solution of $B^{(m)}$ has the same structure as $\Sigma_{u}$, and therefore also the solution for $A^{(m)}$. This particular structure of the model implies a reduction of the number of parameters from 21 to 12. It should be emphasized that unlike the high frequency process, the low frequency process is not characterized by diagonal parameter matrices.

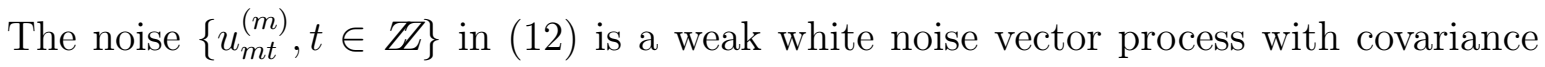
$\operatorname{matrix} \Sigma_{u}^{(m)}$ with

$$
\operatorname{vec}\left(\Sigma_{u}^{(m)}\right)=\left(I_{N^{2}}+B^{(m)} \otimes B^{(m)}\right)^{-1} \operatorname{vec}\left(S_{m}\right) .
$$

Therefore, the parameter matrices of the aggregated process can be obtained from the high frequency process. The matrices $S_{m}$ and $G_{m}$ given by (15) and (16) are functions of the matrices $A, B$, and $\Sigma_{u}$ and thus can be calculated if the high frequency process is known. As for $B^{(m)}$, (14) is a system of nonlinear equations that can not be solved explicitly but for which numerical procedures provide solutions very quickly.

We summarize this section by collecting the main conditional and unconditional moments in Table 1 under the assumption of strong multivariate GARCH with spherically distributed innovations of the high frequency process.

\section{Estimation}

In this section we discuss possible estimation methods for the temporally aggregated multivariate GARCH processes. Certainly the most common estimation method is maximum 
likelihood, which assumes a specific conditional distribution of the errors. In many situations, assuming Gaussian innovations provides consistent maximum likelihood estimates even if this assumption is wrong. In that case we speak of quasi maximum likelihood estimation. In some cases, consistent quasi maximum likelihood estimation under misspecification may also be available assuming non-Gaussian innovations, see Newey and Steigerwald (1997). But for our purpose we restrict QML to the common assumption of Gaussian innovations. In formalizing this approach in the following, we suppress the subscript $(m)$ from all variables and parameters for notational simplicity.

Denote by $\theta$ the parameter vector that characterizes $h_{t}=\operatorname{vech}\left(H_{t}\right)$, the best linear predictor of $\eta_{t}$, i.e., $\theta=\operatorname{vec}(\omega, A, B)$. To stress the dependence of $H_{t}$ on this parameter vector we write it as $H_{t}(\theta)$. Suppose that there is an underlying data generating process characterized by the unknown parameter vector $\theta_{0}$ which one wants to estimate using a given sample of $T$ observations. The quasi maximum likelihood (QML) approach estimates $\theta_{0}$ by maximizing the log likelihood function assuming normality.

Definition 2 (QML) The quasi maximum likelihood estimator is defined by

$$
\hat{\theta}_{Q M L}=\arg \max _{\theta \in \Theta} \sum_{t=1}^{T} l_{t}(\theta)
$$

where

$$
l_{t}(\theta)=-\frac{N}{2} \ln (2 \pi)-\frac{1}{2} \ln \left|H_{t}(\theta)\right|-\frac{1}{2} \varepsilon_{t}^{\prime} H_{t}^{-1}(\theta) \varepsilon_{t}
$$

conditional on some starting value for $H_{0}$.

Bollerslev and Wooldridge (1992) have discussed the properties of QML estimates $\hat{\theta}_{Q M L}$ in a general conditional heteroskedasticity model framework. One of their conditions for consistency is that the first two conditional moments of $\varepsilon_{t}$ are correctly specified. As we have seen from the previous section, however, this is not the case for weak multivariate GARCH models, where the conditional variance can differ from the predictor $H_{t}(\theta)$. More specifically, Jeantheau (1998) assumes a semi-strong multivariate GARCH model to prove consistency of QML, and the same was done by Comte and Lieberman (2003) to prove asymptotic normality.

Of course one can still do estimation and inference using QML, hoping that the difference between $H_{t}(\theta)$ and the true conditional variance is negligible. If the model is correctly 
specified, that is, in the semi-strong and strong case, then QML estimates are asymptotically normally distributed, even in the case of non-normally distributed innovations. The asymptotic distribution is given by

$$
\sqrt{T}\left(\hat{\theta}_{Q M L}-\theta_{0}\right) \stackrel{\mathcal{D}}{\longrightarrow} N\left(0, \mathcal{J}^{-1} \mathcal{I} \mathcal{J}^{-1}\right),
$$

where

$$
\mathcal{I}=\mathrm{E}\left[\left.\frac{\partial l_{t}(\theta)}{\partial \theta} \frac{\partial l_{t}(\theta)}{\partial \theta^{\prime}}\right|_{\theta_{0}}\right], \quad \mathcal{J}=-\mathrm{E}\left[\left.\frac{\partial^{2} l_{t}(\theta)}{\partial \theta \partial \theta^{\prime}}\right|_{\theta_{0}}\right]
$$

and where the expectation is taken with respect to the true process. The matrix $\mathcal{I}$ is the expectation of the outer product of the score vector evaluated at the true parameter vector $\theta_{0}$ and is often called the information matrix, whereas $\mathcal{J}$ is the negative expectation of the Hessian evaluated at $\theta_{0}$. If the error process $\varepsilon_{t}$ is conditionally Gaussian, then $\mathcal{I}=\mathcal{J}$ and the asymptotic covariance matrix reduces to $\mathcal{I}^{-1}$, the Cramer-Rao lower bound.

We can now do QML estimation of the temporally aggregated process by maximizing the quasi log-likelihood (22). Inference is done by using the empirical counterparts of the matrices $\mathcal{I}$ and $\mathcal{J}$, replacing the expectations by sample means, evaluated at $\hat{\theta}_{Q M L}$. These are used for the estimation of the asymptotic covariance matrix in (23).

A second possibility is to use nonlinear least squares (NLS) estimation, as proposed by Francq and Zakoian (2000) for univariate weak GARCH models. The justification for this approach lies in the linear regression form of the VARMA representation (3). Least squares estimation of VAR models is known to be consistent and asymptotically efficient under quite general conditions and if the error distribution is multinormally distributed, see e.g., Lütkepohl (1993). The moving average part complicates the estimation, since no closed form solution to the normal equations are available. However, numerical techniques can be used just as in QML estimation. Since the error term $u_{t}$ of the VARMA representation (3) is not Gaussian, the least squares estimation is likely to be quite inefficient. However, it will still be consistent under mild conditions. One of the objectives of this paper is to assess the empirical performances of both QML and NLS estimates.

Formally, the NLS estimator is defined as follows.

Definition 3 (NLS) The nonlinear least squares estimator is defined by

$$
\hat{\theta}_{N L S}=\arg \min _{\theta \in \Theta} \sum_{t=1}^{T} u_{t}(\theta)^{\prime} u_{t}(\theta)
$$

where

$$
u_{t}(\theta)=\eta_{t}-\omega-(A+B) \eta_{t-1}+B u_{t-1}(\theta)
$$


conditional on some starting values $u_{0}$ and $\eta_{0}$.

In a univariate framework, Francq and Zakoian (2000) show that $\hat{\theta}_{N L S}$ is consistent and asymptotically normally distributed under mild conditions and proposes a method to estimate the asymptotic covariance matrix.

When the data of the high frequency process are available it is of course possible to first estimate the high frequency parameters by maximum likelihood and then transform the estimates according to the above functions to obtain parameter estimates of the aggregated series. It is then also possible via the delta method to obtain the asymptotic covariance matrix of the low frequency parameter estimates by using the corresponding matrix of the high frequency estimates and the nonlinear functions that link the former to the latter. Standard errors are likely to be smaller when high frequency data are used, as more observations are used for estimation.

\section{A simulation study}

In this section we investigate the properties of the QML and NLS estimators applied to temporally aggregated data coming from a high frequency multivariate GARCH process. The parameters of the high frequency process are chosen such that they resemble typical values for financial data, see for example the empirical analysis in Section 6. We study the behavior of the two estimators for different levels of aggregation using a bivariate $\operatorname{GARCH}(1,1)$ model.

\subsection{The high frequency process}

The high frequency process $(m=1)$ is assumed to be a strong MGARCH process. We choose a diagonal VEC model with innovations that are generated either from a bivariate normal distribution or from a bivariate student $t$ distribution with 8 degrees of freedom. This allows us to assess the effect of alternative kurtosis and co-kurtosis coefficients on the parameter estimates. The parametrization of the process is as follows.

$$
\begin{aligned}
\varepsilon_{t} & =H_{t}^{1 / 2} \xi_{t} \\
\operatorname{vech}\left(H_{t}\right)=h_{t} & =\left[\begin{array}{c}
1 \\
\omega_{2} \\
1
\end{array}\right]+\left[\begin{array}{ccc}
0.07 & 0 & 0 \\
0 & 0.08 & 0 \\
0 & 0 & 0.085
\end{array}\right] \eta_{t-1}+\left[\begin{array}{ccc}
0.9 & 0 & 0 \\
0 & 0.9 & 0 \\
0 & 0 & 0.9
\end{array}\right] h_{t-1}(27)
\end{aligned}
$$


where

$$
\begin{aligned}
\xi_{t} & \sim i . i . d . N\left(0, I_{2}\right) \\
\xi_{t} & \sim i . i . d . t_{8}\left(0, I_{2}\right)
\end{aligned}
$$

and where $\omega_{2}=\rho \sqrt{200} / 15$ and $\rho$ is the unconditional correlation coefficient. We choose five alternative values for $\rho$ : $0,0.1,0.3,0.5$, and 0.7 . The sample size is chosen to be $T=5000$. The process in (26) and (27) is stationary with maximum eigenvalue of $A+B$ equal to 0.985. The unconditional variances of $\varepsilon_{t}$ are given by 33.33 and 66.66 , respectively. In the case of Gaussian $\left(t_{8}\right)$ innovations, the unconditional kurtosis of $\varepsilon_{1 t}$ is 3.60 (6.34), that of $\varepsilon_{2 t}$ is 5.83 (29.86) and the unconditional co-kurtosis is 1.46 (3.26). Let us first consider the case $\rho=0$. While the unconditional covariance is zero in this case, the conditional covariance fluctuates around zero. By restricting $\rho$ to zero in the diagonal vec model, the estimation problem using the aggregated data involves 8 parameters less than in the case where $\rho$ is different from zero. Therefore we present detailed results for the case $\rho=0$ and summary results for the other cases.

Table 2 reports the results for the high frequency model. Results using the multivariate $t$ distribution are not given here to economize on space, but they are included in Hafner and Rombouts (2003). The process in (26) and (27) is generated 500 times and then estimated by QML and NLS. Reported are summary statistics of the distribution of parameter estimates, as well as the total mean squared error (MSE), i.e., the trace of the mean squared error matrix. Comparing the QML results for normal innovations with $t_{8}$ innovations, the bias is very similar but the standard deviations are higher for the $t_{8}$ innovations, giving a slightly higher mean squared error (MSE). This is at least partly due to the fact that QML is inefficient in this case. There are small differences between the biases measured by the mean and the median for the QML method, showing that the finite sample distribution of QML parameter estimates is slightly asymmetric. In the tables, significant deviations of the mean from the true value are indicated with asterisks, one for the $5 \%$ level and two for the $1 \%$ level. As the high frequency process is generated as strong GARCH, it may be somewhat surprising that QML estimates of the parameters in $\omega$ and $B$ are significantly different from the true values. However, note that the median is slightly closer to the true values. Given that asymptotically median and mean should be the same (because the asymptotic distribution is normal), we conjecture that our sample size may not suffice to obtain unbiased QML estimates. It should also be emphasized that estimation of bivariate GARCH models is by far more complicated than that of univariate GARCH models, so 
the small absolute values of the biases should be satisfactory from a practical viewpoint.

As expected, the nonlinear least squares results are decidedly worse than the quasi maximum likelihood results for both the normal and the student $t$ innovations, due to the inefficiency as outlined in the previous section. The parameter estimates of the $A$ and $B$ matrices have a reasonably low bias, but the constants $\omega_{1}$ and $\omega_{2}$ are systematically overestimated. This overestimation of the constants in the conditional variance matrix interacts with the underestimation of $b_{11}$ and $a_{33}$ for respectively the first and second constant. We also perform a simulation exercise, the results of which are not reported here, using the variance targeting technique of Engle and Mezrich (1996), which means that we fix $\omega=\left(I_{3}-A-B\right) \hat{\sigma}$ where $\hat{\sigma}$ contains the empirical variances and the covariance of the high frequency data. We do this to evaluate whether the NLS biases of $b_{11}$ and $a_{33}$ are somewhat reduced, but the results did not change substantially. The high standard deviations of the NLS estimates indicate the inefficiency of this method. As a consequence, the total mean square errors are much higher than those of QML, 2.1262 and 3.0805 for the normal case and the student $t$ case respectively. The difference between the total MSE's is larger than for the QML estimator, i.e. the NLS estimator is more sensitive to the kurtosis of the innovations. The parameter space for the NLS estimation is restricted to avoid extreme values for the parameter estimates. For example $\omega_{1}$ and $\omega_{3}$ are restricted to be smaller than 6 which can be noticed by inspection of the maxima in the NLS panels. The distribution of the NLS parameter estimates is in most cases quite asymmetric, which can be seen by the difference between the mean and median. The median bias is larger than the mean bias for the $A$ parameters but smaller for $\omega$ and $B$, which holds for both normal and student t innovations.

We now turn to the case $\rho \neq 0$. Rather than reporting estimation results for the nine parameters, Table 5 reports the mean square errors of the one-step predictions of conditional variances and conditional covariance. This measure has also been used by Francq and Zakoian (2000) in the univariate setting to evaluate the relative forecasting performance of QML and NLS. We find that in general, QML has a smaller mean square prediction error. Taking into account the standard error of the simulations, which we do not report to economize on space, the difference between QML and NLS is not significant. Note that the MSPE decreases for the conditional variances and increases for the conditional covariance when $\rho$ is increased. 


\subsection{Low frequency processes}

For the aggregation level of the low frequency process, we consider two cases: $m=2$ and $m=10$. The true parameters of the aggregated process are obtained by (13), (14) and (17). In order to obtain the same number of observations of the low frequency process whatever the value of $m$, we generate $T=5000 \mathrm{~m}$ observations of the high frequency process. As mentioned above, the scenario $\rho=0$ of the high frequency process allows us to estimate also a restricted VEC model for the low frequency process, although not a diagonal one. Recall that even if the high frequency parameter matrices are diagonal, the low frequency parameters are in general not diagonal anymore. In other words, the class of diagonal VEC models is not closed under temporal aggregation. Thus, the model we estimate for the case $\rho=0$ is given by

$$
h_{m t}^{(m)}=\left[\begin{array}{c}
\omega_{1}^{(m)} \\
0 \\
\omega_{3}^{(m)}
\end{array}\right]+\left[\begin{array}{ccc}
a_{11}^{(m)} & 0 & a_{13}^{(m)} \\
0 & a_{22}^{(m)} & 0 \\
a_{31}^{(m)} & 0 & a_{33}^{(m)}
\end{array}\right] \eta_{m(t-1)}^{(m)}+\left[\begin{array}{ccc}
b_{11}^{(m)} & 0 & b_{13}^{(m)} \\
0 & b_{22}^{(m)} & 0 \\
b_{31}^{(m)} & 0 & b_{33}^{(m)}
\end{array}\right] h_{m(t-1)}^{(m)} .
$$

Note that the solution for $\omega_{i}^{(m)}, a_{i j}^{(m)}$ and $b_{i j}^{(m)}$ depends on the innovation distribution, i.e. the true parameter values are different when we switch from normal innovations to student $t$ innovations.

Table 3 presents the results of the simulation exercise for $m=2$. The initial data of the high frequency process are divided by $\sqrt{m}$ in order to obtain series with the same unconditional variance. For the diagonal elements in (28), the QML method still yields reasonably small biases but larger standard deviations compared with $m=1$, for both normal innovations and student $t$ innovations. The true off-diagonal elements of the aggregated model are close to zero and the QML biases and standard deviations for these parameters are still quite small. Again, there are tiny differences between the mean bias and the median bias for the QML method, indicating that the parameter distribution is almost symmetric. Overall, the median bias tends to be smaller than the mean bias. Generally speaking, QML still works well in the setting with $m=2$. The total MSE is 0.6109 and 0.4790 for the normal case and the student $t$ case respectively.

The results for NLS and $m=2$ show that some parameters such as $b_{11}^{(2)}$ and $a_{33}^{(2)}$ are underestimated, which corresponds to the large biases for $\omega_{1}^{(2)}$ and $\omega_{2}^{(2)}$, respectively. ${ }^{2}$ The

\footnotetext{
${ }^{2}$ In GARCH type models, the constant is typically negatively correlated with the $\alpha$ and with the $\beta$ parameter. This is plausible from the formula for the unconditional variance, which should match the empirical variance, a given value.
} 
inefficiency of NLS is again clearly demonstrated by the large standard deviations. Unlike QML, some NLS parameter estimates are not symmetrically distributed, and again the median biases of the parameters in $\omega$ and $B$ are smaller than the mean biases, but that of $A$ is larger. The difference in total MSE between normal (8.2043) and student $t$ (52.8210) innovations is quite pronounced. As for $m=1$, variance targeting does not improve the results. Thus, for $m=2$ one would also clearly prefer QML over NLS, as the latter does not help in reducing the finite sample bias but has a much higher variation.

Turning to $m=10$, Table 4 summarizes the results. A general impression is that QML biases become more pronounced compared to the $m=2$ case, especially for the aggregated $b_{i i}^{(10)}$ parameters. The underestimation of the parameters in $B$ and the overestimation of those in $\omega$ corresponds exactly to the results reported by Meddahi and Renault (2004) for the univariate case. Also, the variation of the parameter estimates rises importantly.

The least squares results are comparable to the $m=2$ case with often a smaller median bias than mean bias. However, the total MSE's are still more than ten times larger than those of QML. In any practical situation one would clearly prefer to use QML estimation.

We also examined the case $m=50$ for which the results are not reported here. The main conclusions for this case are that neither QML nor NLS perform satisfactorily. Both methods provide intolerable biases and standard deviations. Furthermore, it happens frequently that convergence of the estimation algorithm can not be achieved. We can therefore conclude that in our setting QML outperforms NLS, but for large $m$ QML seems to be biased for the parameter matrix $B$. This shows that the conclusion of Meddahi and Renault (2004) of significantly biased QML estimates at large aggregation levels carries over to the multivariate case. Given the conjectured consistency of NLS (extending the results of Francq and Zakoian (2000) to the multivariate case), one would expect a critical sample size above which NLS is preferable to QML, provided that QML is inconsistent. But even if QML is inconsistent, the critical sample size above which NLS should be preferred is likely to be so large that NLS will be irrelevant in most practical situations.

Finally, Table 5 reports results of the mean square prediction errors for alternative values of $\rho$ and $m=2$. Comparing QML and NLS, the result is that on average QML outperforms NLS, but taking into account the variation across the 500 replications, the difference between the two methods is again not significant. Thus, we cannot conclude that NLS provides superior predictions in terms of mean square prediction error. 


\section{Empirical example}

In this section we present an application to a bivariate stock index series. We use the Dow Jones Industrial Average index (DOW) and the NASDAQ index from January 1980 to June 2003. Summary statistics of the log returns are provided in Table 6 . Note that the NASDAQ has a higher standard deviation but a lower skewness and kurtosis. The skewness and kurtosis are strongly affected by the 1987 crash, where the DOW fell stronger than the NASDAQ. This holds for daily, weekly, and bi-weekly returns.

We estimated a diagonal VEC model for two aggregation levels. In theory, some offdiagonal elements of the parameter matrices of the aggregated model should be non-zero, but these were found to be very small and insignificant. QML estimation results are reported in Table 7 .

Figure 1 plots the estimated conditional correlations and volatilities using the daily series, whereas Figures 2 and 3 do the same for the weekly and bi-weekly series. In each figure, the upper panel shows the estimated conditional correlation and the lower panel the estimated conditional variances. Consider first the conditional correlations. For most of the time until about 1993, the correlation between DOW and NASDAQ was on a high level around 0.8. After that it drops somewhat, where the drop is more pronounced for the weekly and bi-weekly returns than for the daily returns. The biggest change occurs in 2000, where correlation drops to zero for all considered aggregation levels, and then slowly increases again to attain the pre-2000 level. This is due to the decoupling of the two markets during the new economy boom, where NASDAQ stock prices increased much stronger than DOW stock prices.

Looking at the estimated conditional variances, one notes the enormous effect of the October 1987 crash in the DOW, which was remarkably smaller in the NASDAQ. Inversely, the NASDAQ was much more volatile than the DOW in the years of the new economy boom, i.e., 1996 to 2000. This is apparent in daily returns, but even more so in weekly and bi-weekly returns.

Also reported in Table 7 are the parameters of $A^{(m)}+B^{(m)}$ implied by the estimation of the high frequency process, based on equation (17). That is, we calculate $(A+B)^{5}$ and $(A+B)^{10}$ to obtain the weekly and bi-weekly parameters implied by the daily estimates, and further calculate $\left(A^{(5)}+B^{(5)}\right)^{2}$ to obtain the bi-weekly parameters implied by the weekly estimates. Note that for the diagonal VEC model used here for the high frequency process, all matrices $A^{(m)}+B^{(m)}$ of the aggregated process are diagonal as well. The 
eigenvalues of this matrix are just given by the diagonal elements, and the largest of these is often considered as a coefficient measuring persistence. It is striking from the results in Table 7 that the daily estimates imply low frequency models with smaller persistence than the estimates of the low frequency models. On the other hand, the weekly estimates

imply bi-weekly persistence that closely matches the persistence estimated with bi-weekly data. In other words, estimation results using daily data on the one hand and weekly and bi-weekly data on the other hand suggest that the model used is not coherent with respect to the sampling frequency, whereas only considering weekly and bi-weekly data it appears to be coherent. This hints at a possible mis-specification of the model used for daily data. It also confirms previous results, e.g. by Andersen and Bollerslev (1997), that financial time series tend to become more complicated and different from simple GARCH models if the sampling frequency is increased.

\section{Conclusions and Outlook}

We have investigated the performance of two estimation techniques, quasi maximum likelihood (QML) and nonlinear least squares (NLS), for temporally aggregated multivariate GARCH models. In a simulation study, we draw the main conclusion that for small aggregation levels one would prefer to use QML due to the higher efficiency relative to NLS. We could not find any strong bias in the QML estimates for typical example processes, allowing for alternative unconditional correlations, and NLS estimation did not reduce the bias substantially. For very large aggregation levels neither method performed well and convergence of the optimization algorithms became a non-trivial task. We conjecture that this is due to the remaining large kurtosis after temporal aggregation, where the dynamics are already close to white noise. We have also tried semiparametric estimation methods as in Hafner and Rombouts (2004), but this did not improve the results for QML. Altogether, our study suggests that in most practical situations, there will be no better choice than QML for parameter estimation. 


\section{Acknowledgments}

The authors would like to thank Luc Bauwens and Feike Drost for helpful discussions.

This text presents research results of the Belgian Program on Interuniversity Poles of Attraction initiated by the Belgian State, Prime Minister's Office, Science Policy Programming. The scientific responsibility is assumed by the authors.

\section{References}

Andersen, T., And T. Bollerslev (1997): "Intraday Periodicity and Volatility Persistence in Financial Markets," Journal of Empirical Finance, 4, 115-158.

Bauwens, L., S. Laurent, and J. Rombouts (2003): "Multivariate GARCH Models: A Survey," CORE DP 2003/31.

Bollerslev, T., And J. Wooldridge (1992): "Quasi-Maximum Likelihood Estimation and Inference in Dynamic Models with Time-varying Covariances," Econometric Reviews, 11, 143-172.

Comte, F., and O. Lieberman (2003): "Asymptotic Theory for Multivariate GARCH Processes," Journal of Multivariate Analysis, 84, 61-84.

Drost, C., And T. Nijman (1993): "Temporal Aggregation of GARCH Processes," Econometrica, 61, 909-927.

Engle, R., And J. MEZRich (1996): "GARCH for Groups," RISK, 9, 36-40.

FrancQ, R., And J.-M. Zakoian (2000): "Estimating Weak GARCH Representations," Econometric Theory, 16, 692-728.

HAFner, C. (2003): "Fourth Moment Structure of Multivariate GARCH Processes," Journal of Financial Econometrics, 1, 26-54.

- (2004): "Temporal Aggregation of Multivariate GARCH Processes," Econometric Institute Report 29, Erasmus University Rotterdam.

Hafner, C., And J. Rombouts (2003): "Estimation of Temporally Aggregated Multivariate GARCH Models," CORE DP 2003/73. 
(2004): "Semiparametric Multivariate Volatility Models," Econometric Institute Report 21, Erasmus University Rotterdam.

Jeantheau, T. (1998): "Strong Consistency of Estimators for Multivariate ARCH models," Econometric Theory, 14, 70-86.

Lütkepohl, H. (1993): Introduction to Multiple Time Series Analysis. Springer-Verlag.

Meddahi, N., And E. Renault (2004): "Temporal Aggregation of Volatility Models," forthcoming in Journal of Econometrics.

Newey, W., And D. Steigerwald (1997): "Asymptotic Bias of Quasi Maximum Likelihood Estimators in Conditional Heteroskedasticity Models," Econometrica, 65, 587-599. 
Table 1: Summary of moments of high and low frequency processes

\begin{tabular}{cccccc}
\hline \hline$x_{t}$ & $E_{m(t-1)}\left(x_{t}\right)$ & $E\left(x_{t}\right)$ & $\operatorname{vech}\left(\operatorname{Var}_{m(t-1)}\left(x_{t}\right)\right)$ & $\operatorname{Var}\left(x_{t}\right)$ & $\operatorname{Cov}\left(x_{m t}, x_{m(t-\tau)}\right)$ \\
\hline$\varepsilon_{t}$ & 0 & 0 & $h_{t}$ & $\Sigma_{\varepsilon}$ & 0 \\
$u_{t}$ & 0 & 0 & $\left(c \mathcal{G}_{K}-I_{N^{2}}\right)\left(h_{t} \otimes h_{t}\right)$ & $\Sigma_{u}$ & 0 \\
$\eta_{t}$ & $h_{t}$ & $\sigma$ & $\left(c \mathcal{G}_{K}-I_{N^{2}}\right)\left(h_{t} \otimes h_{t}\right)$ & $\Gamma_{\eta}(0)$ & $\Gamma_{\eta}(\tau)$ \\
$h_{t}$ & $h_{t}$ & $\sigma$ & 0 & $\Gamma_{h}(0)$ & $\Gamma_{h}(\tau)$ \\
\hline & & & & $m \Sigma_{\varepsilon}$ & 0 \\
$\varepsilon_{m t}^{(m)}$ & 0 & 0 & $*$ & $\Sigma_{u}^{(m)}$ & 0 \\
$u_{m t}^{(m)}$ & 0 & 0 & $*$ & $\Gamma_{\eta}^{(m)}(0)$ & $\Gamma_{\eta}^{(m)}(\tau)$ \\
$\eta_{m t}^{(m)}$ & $(29)$ & $m \sigma$ & $*$ & $\Sigma_{w}^{(m)}$ & 0 \\
$w_{m t}^{(m)}$ & 0 & 0 & $* 9)$ & $*$ \\
\hline \hline
\end{tabular}

The $*$-sign indicates that these expressions can be derived following the same type of calculations of Hafner (2003a). The matrix $\mathcal{G}_{K}$ is given by $2\left(D_{K}^{+} \otimes D_{K}^{+}\right)\left(I_{K} \otimes C_{K K} \otimes\right.$ $\left.I_{K}\right)\left(D_{K} \otimes D_{K}\right)+I_{N^{2}}$. The scalar $c$ is the cokurtosis of the innovations, $D_{K}$ is the duplication matrix, $D_{K}^{+}$its generalized inverse and $C_{K K}$ the commutation matrix. See Lütkepohl (1996) for definitions of these matrices. Expression (29) is given by

$E_{m(t-1)}\left[\eta_{m t}^{(m)}\right]=(I-A-B)^{-1}\left(I-(A+B)^{m}\right) h_{m(t-1)+1}+\sum_{i=0}^{m-2}(m-1-i)(A+B)^{i} \omega(29)$

where the second term of (29) is zero for $m=1$. 


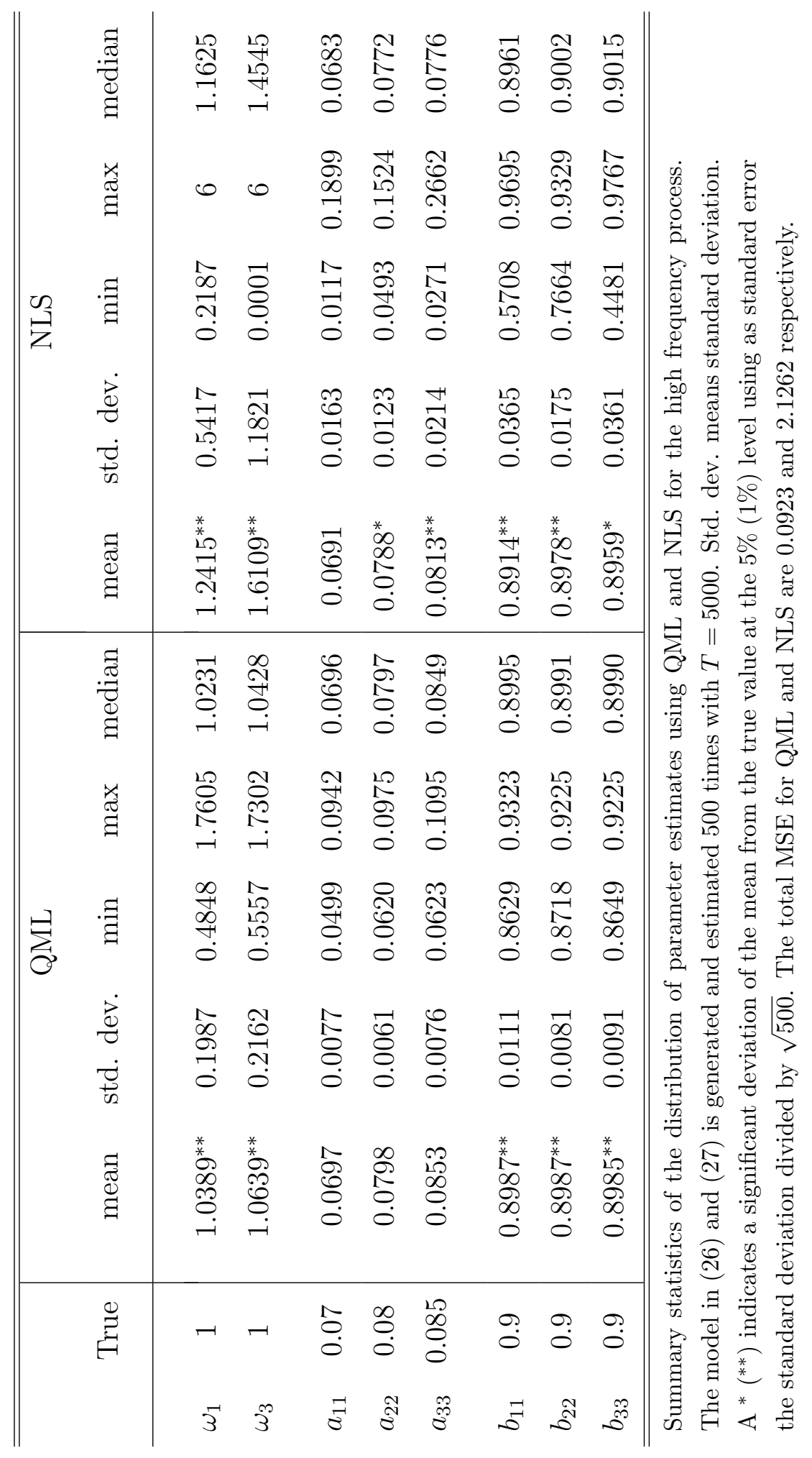

Table 2: Simulation results for $m=1$ with normal innovations 


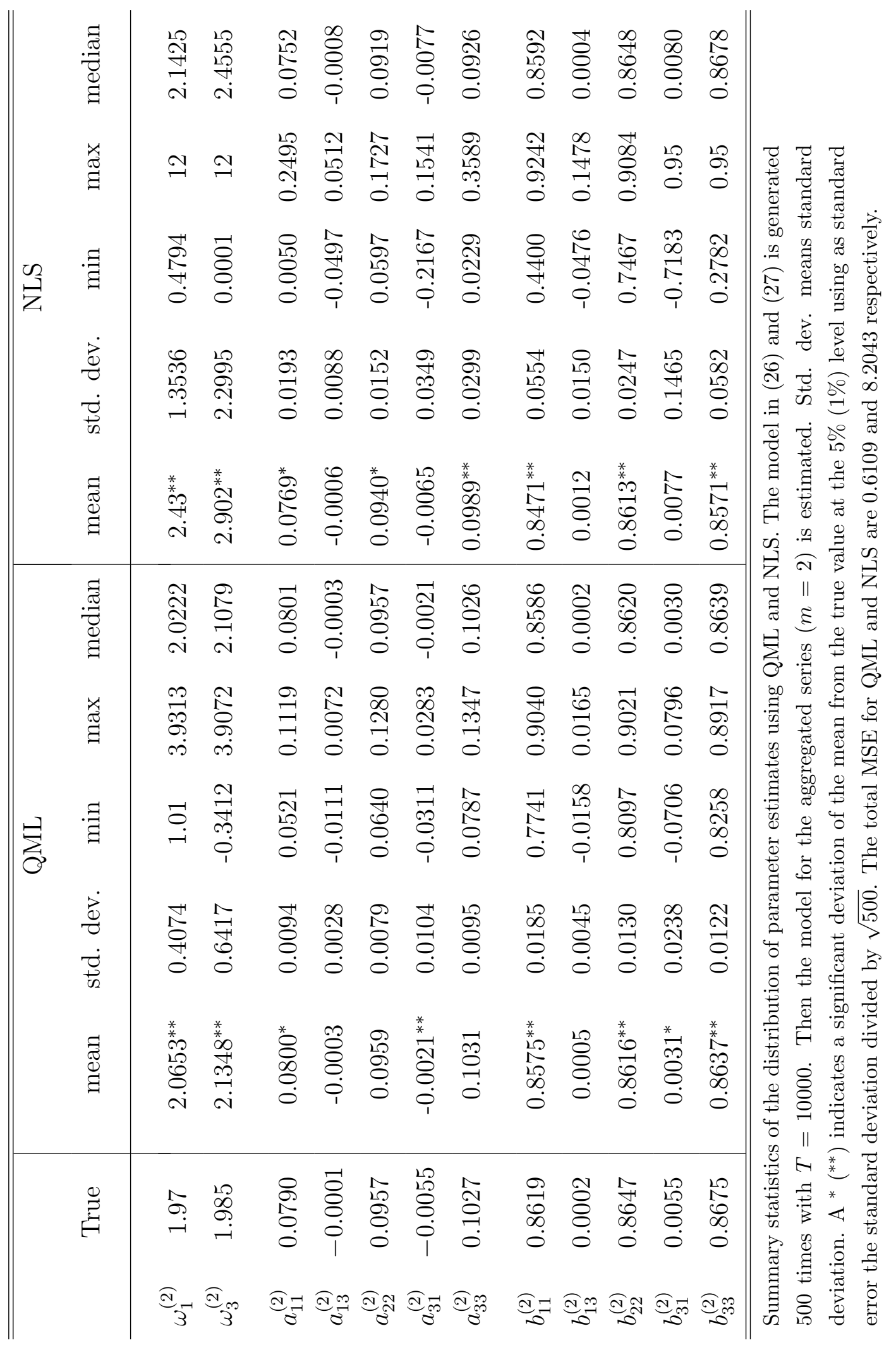

Table 3: Simulation results for ${ }^{2} \frac{1}{m}=2$ with normal innovations 


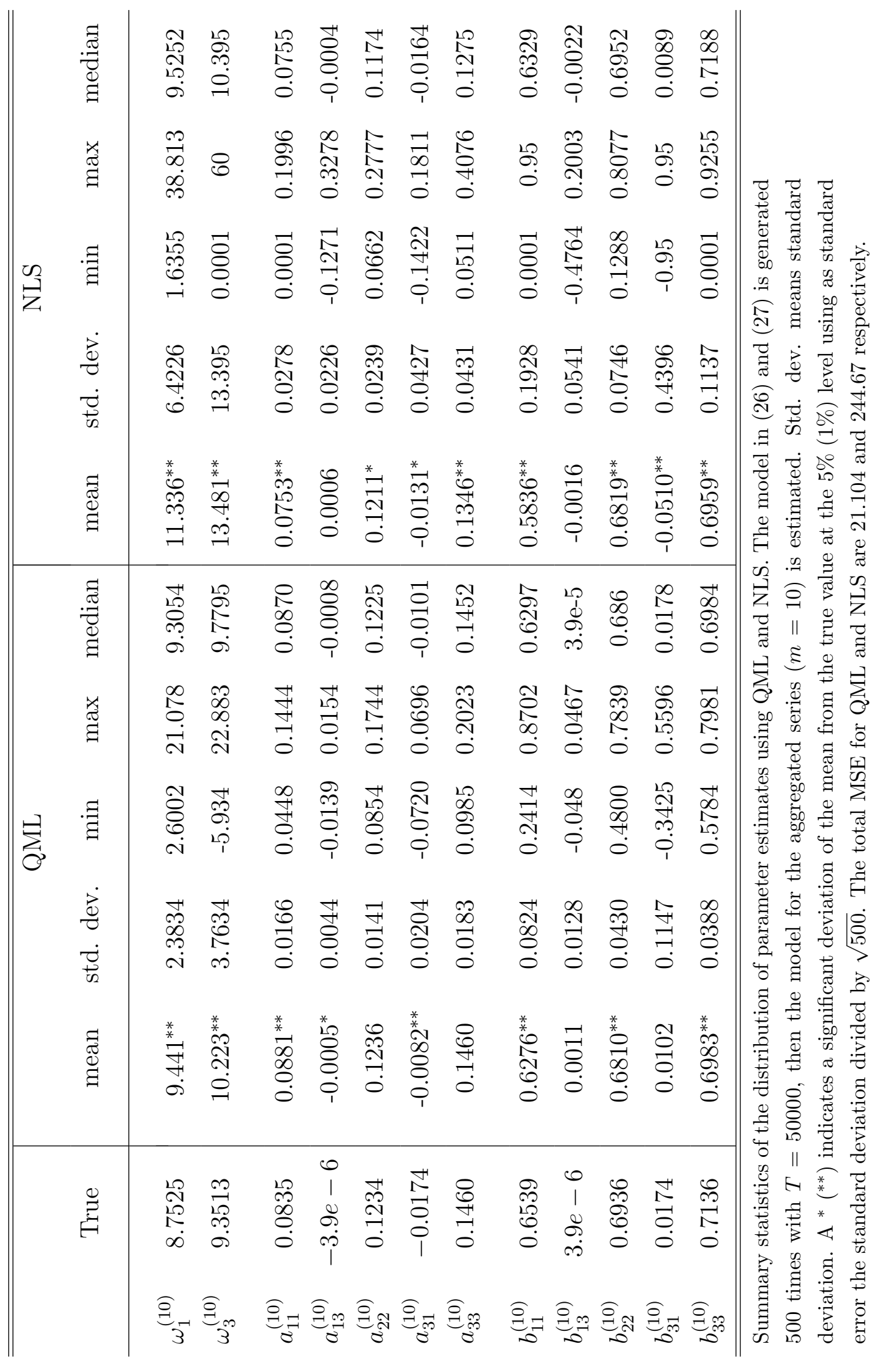

Table 4: Simulation results for $m=10$ with normal innovations 
Table 5: Mean squared prediction errors

\begin{tabular}{|c|c|c|c|c|c|c|}
\hline \multirow{2}{*}{$\begin{array}{c}m=1 \\
\rho\end{array}$} & \multicolumn{3}{|c|}{ QML } & \multicolumn{3}{|c|}{ NLS } \\
\hline & $h_{11}$ & $h_{12}$ & $h_{22}$ & $h_{11}$ & $h_{12}$ & $h_{22}$ \\
\hline 0.1 & 2604 & 2716 & 15060 & 2607 & 2732 & 15347 \\
\hline 0.3 & 2580 & 2907 & 14689 & 2591 & 2941 & 14928 \\
\hline 0.5 & 2540 & 3337 & 14369 & 2542 & 3357 & 14348 \\
\hline 0.7 & 315 & 3476 & 11737 & 2343 & 3573 & 12166 \\
\hline$m=2$ & & QML & & & NLS & \\
\hline$\rho$ & $h_{11}$ & $h_{12}$ & $h_{22}$ & $h_{11}$ & $h_{12}$ & $h_{22}$ \\
\hline 0.1 & 2942 & 3008 & 17328 & 2973 & 3074 & 17601 \\
\hline 0.3 & 2935 & 3278 & 17196 & 2950 & 3304 & 17065 \\
\hline 0.5 & 2870 & 3704 & 16490 & 2912 & 3775 & 16850 \\
\hline 0.7 & 2641 & 4032 & 14269 & 2678 & 4117 & 14562 \\
\hline \multicolumn{7}{|c|}{$\begin{array}{l}\text { Mean squared errors of one-step predictions of conditional } \\
\text { variances and covariances for alternative unconditional cor- } \\
\text { relations } \rho \text { and for the high frequency }(m=1) \text { and low } \\
\text { frequency }(m=2) \text { processes. The reported values are av- } \\
\text { erages over } 500 \text { replications of our bivariate VEC example } \\
\text { process. }\end{array}$} \\
\hline
\end{tabular}


Table 6: Summary statistics

\begin{tabular}{lrrr}
\hline \hline & $m=1$ & $m=5$ & $m=10$ \\
\cline { 1 - 1 } & \multicolumn{2}{c}{ Dow Jones returns } \\
Observations & 6110 & 1222 & \\
Mean & 0.00039 & 0.00193 & 0.00387 \\
Standard Deviation & 0.01078 & 0.02416 & 0.03291 \\
Maximum & 0.09666 & 0.1195 & 0.10746 \\
Minimum & -0.25632 & -0.30924 & -0.32524 \\
Skewness & -2.3318 & -1.7404 & -1.8541 \\
Kurtosis & 60.146 & 26.348 & 19.865 \\
& & & \\
& & & \\
Observations & 6110 & 1222 & 611 \\
Mean & 0.00039 & 0.00195 & 0.00389 \\
Standard Deviation & 0.01327 & 0.03170 & 0.04385 \\
Maximum & 0.13255 & 0.12725 & 0.15141 \\
Minimum & -0.14002 & -0.19518 & -0.21701 \\
Skewness & -0.41565 & -0.95124 & -0.47162 \\
Kurtosis & 14.296 & 8.1694 & 5.3898 \\
\hline \hline
\end{tabular}

Descriptive statistics for the Dow Jones returns and NASDAQ returns from $02 / 01 / 1980$ to $10 / 06 / 2003$ on a daily, weekly and bi-weekly basis. 
Table 7: Diagonal VEC estimates for different time aggregation frequencies

\begin{tabular}{|c|c|c|c|c|c|c|}
\hline & \multicolumn{2}{|c|}{ Daily $(m=1)$} & \multicolumn{2}{|c|}{ Weekly $(m=5)$} & \multicolumn{2}{|c|}{ Bi-weekly $(m=10)$} \\
\hline & Coefficient & Std error & Coefficient & Std error & Coefficient & Std error \\
\hline$\omega_{11}^{(m)}$ & 0.017145 & $(0.00469)$ & 0.120609 & $(0.05622)$ & 0.530121 & $(0.28656)$ \\
\hline$\omega_{21}^{(m)}$ & 0.011888 & $(0.00267)$ & 0.097031 & $(0.03687)$ & 0.316104 & $(0.22101)$ \\
\hline$\omega_{22}^{(m)}$ & 0.015078 & $(0.02416)$ & 0.174297 & $(0.06415)$ & 0.452082 & $(0.21156)$ \\
\hline$\alpha_{11}^{(m)}$ & 0.071864 & $(0.01740)$ & 0.069882 & $(0.01263)$ & 0.091380 & $(0.04568)$ \\
\hline$\alpha_{22}^{(m)}$ & 0.070409 & $(0.01154)$ & 0.063831 & $(0.00990)$ & 0.065885 & $(0.03219)$ \\
\hline$\alpha_{33}^{(m)}$ & 0.095909 & $(0.01164)$ & 0.093720 & $(0.01914)$ & 0.089211 & $(0.02807)$ \\
\hline$\beta_{11}^{(m)}$ & 0.915186 & $(0.01645)$ & 0.916838 & $(0.02023)$ & 0.872395 & $(0.04961)$ \\
\hline$\beta_{22}^{(m)}$ & 0.914904 & $(0.01160)$ & 0.922681 & $(0.01645)$ & 0.907624 & $(0.04174)$ \\
\hline$\beta_{33}^{(m)}$ & 0.893063 & $(0.01168)$ & 0.890637 & $(0.02485)$ & 0.886542 & $(0.03237)$ \\
\hline$\alpha_{11}^{(m)}+\beta_{11}^{(m)}$ & 0.98897 & & 0.98672 & & 0.97575 & \\
\hline$\alpha_{22}^{(m)}+\beta_{22}^{(m)}$ & 0.98705 & & 0.98651 & & 0.97351 & \\
\hline$\alpha_{33}^{(m)}+\beta_{33}^{(m)}$ & 0.98531 & & 0.98435 & & 0.96377 & \\
\hline$\left(\alpha_{11}^{(1)}+\beta_{11}^{(1)}\right)^{m}$ & & & 0.94605 & & 0.89502 & \\
\hline$\left(\alpha_{22}^{(1)}+\beta_{22}^{(1)}\right)^{m}$ & & & 0.93434 & & 0.87779 & \\
\hline$\left(\alpha_{33}^{(1)}+\beta_{33}^{(1)}\right)^{m}$ & & & 0.92416 & & 0.86244 & \\
\hline$\left(\alpha_{11}^{(5)}+\beta_{11}^{(5)}\right)^{2}$ & & & & & 0.97361 & \\
\hline$\left(\alpha_{22}^{(5)}+\beta_{22}^{(5)}\right)^{2}$ & & & & & 0.97320 & \\
\hline$\left(\alpha_{33}^{(5)}+\beta_{33}^{(5)}\right)^{2}$ & & & & & 0.96894 & \\
\hline
\end{tabular}

Std error means the QML standard error. 

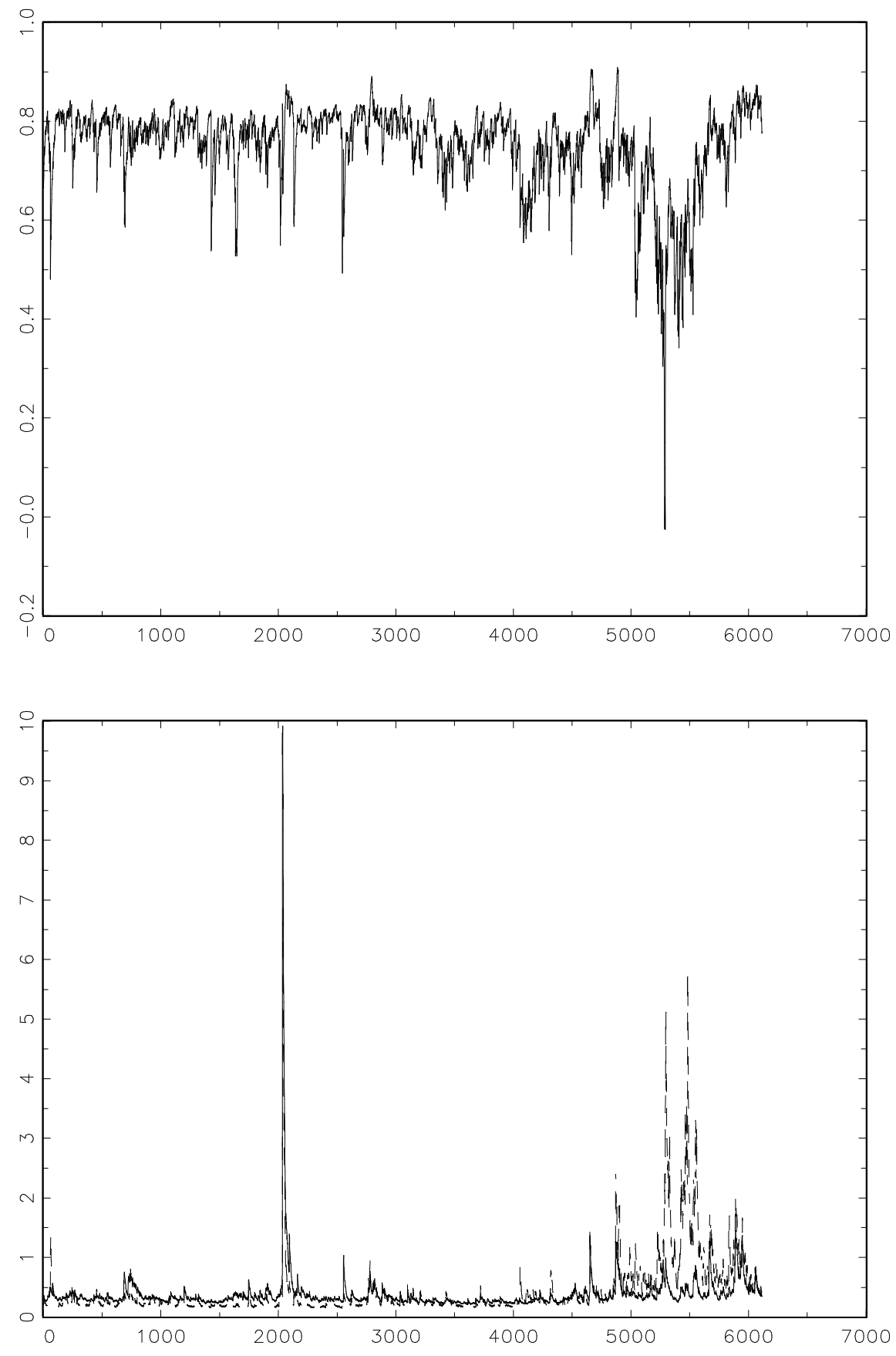

Figure 1: Estimated daily conditional correlations and variances of the Dow Jones and NASDAQ from 02/01/1980 to 10/06/2003. Solid line: Dow Jones. Dashed line: NASDAQ 

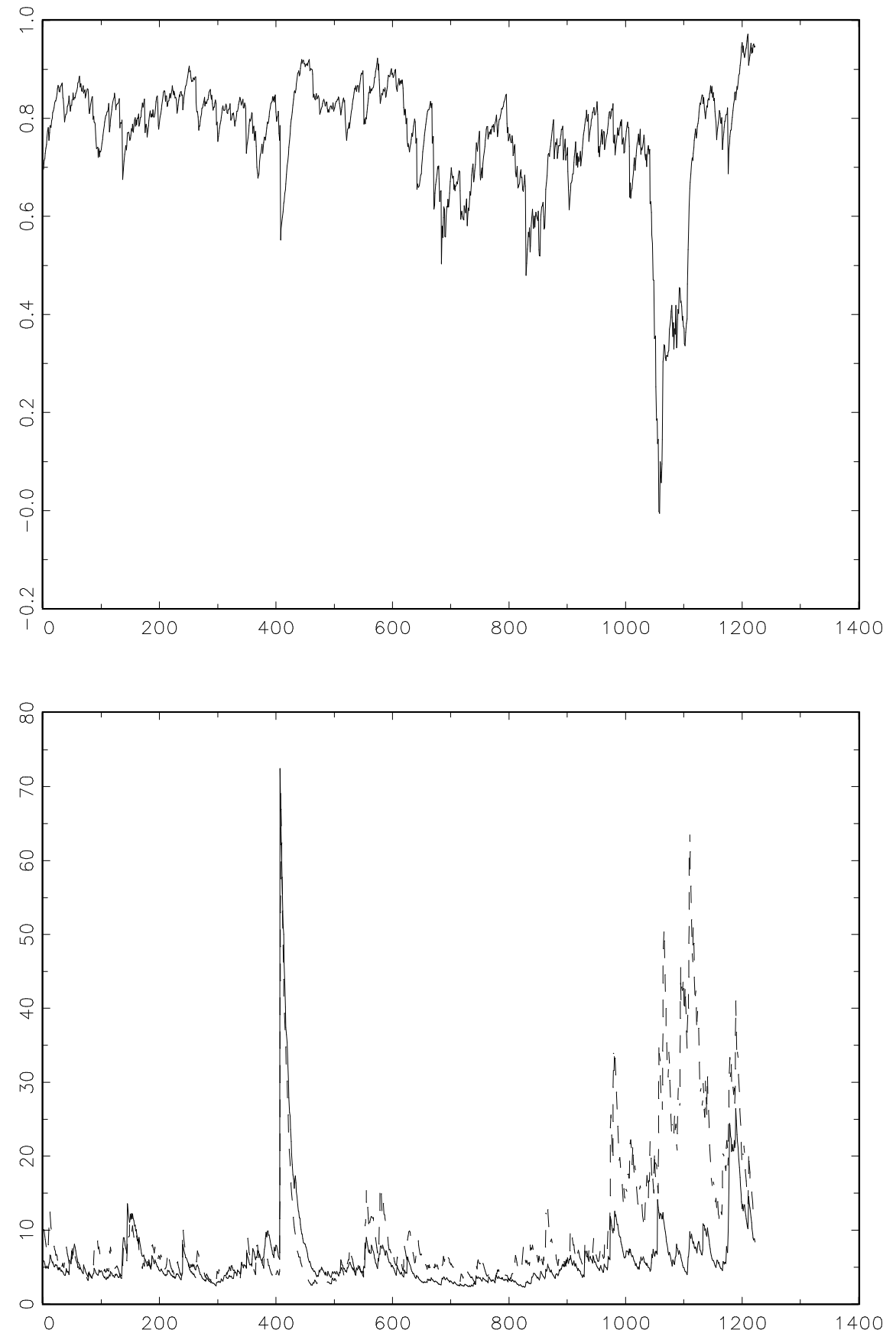

Figure 2: Estimated weekly conditional correlations and variances of the Dow Jones and NASDAQ from 02/01/1980 to 10/06/2003. Solid line: Dow Jones. Dashed line: NASDAQ 

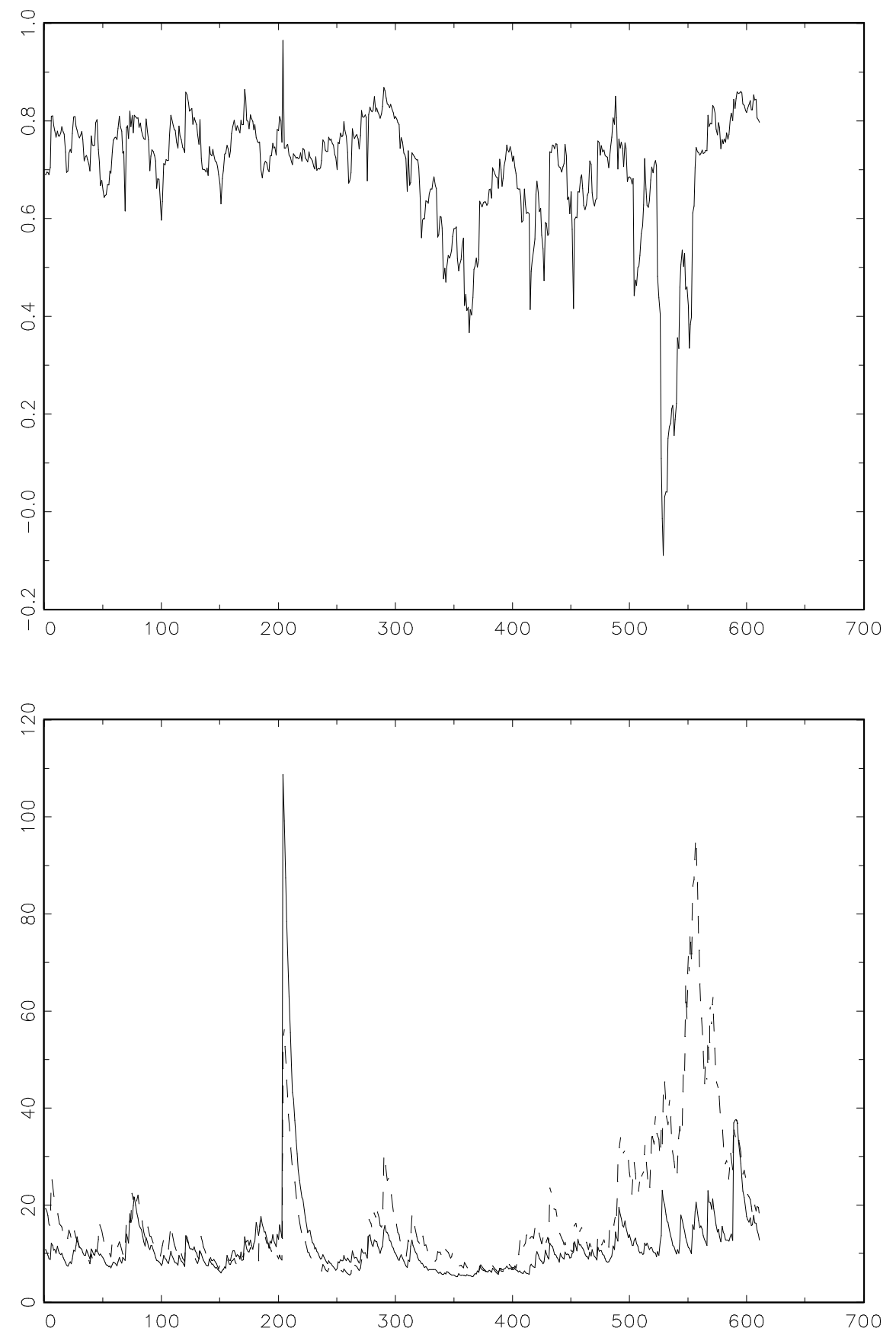

Figure 3: Estimated bi-weekly conditional correlations and variances of the Dow Jones and NASDAQ from 02/01/1980 to 10/06/2003. Solid line: Dow Jones. Dashed line: NASDAQ 